\title{
A Nosé-Hoover Thermostat Adapted to a Slab Geometry
}

\author{
Sz. Maćkowiak ${ }^{1}$, S. Pieprzyk ${ }^{2}$, A.C. Brańka ${ }^{2}$, D.M. Heyes ${ }^{3}$ \\ ${ }^{1}$ Institute of Physics, Poznań University of Technology \\ Piotrowo 3, 60-965 Poznań, Poland \\ E-mail:szymon.mackowiak@put.poznan.pl \\ ${ }^{2}$ Institute of Molecular Physics, Polish Academy of Sciences \\ M. Smoluchowskiego 17, 60-179 Poznań, Poland \\ E-mail: pieprzyk@ifmpan.poznan.pl,branka@ifmpan.poznan.pl \\ ${ }^{3}$ Department of Physics, Royal Holloway \\ University of London, Egham, Surrey TW20 OEX, UK \\ E-mail:david.heyes@rhul.ac.uk
}

Received: 01 December 2016; revised: 22 February 2017; accepted: 27 February 2017; published online: 06 April 2017

\begin{abstract}
A Nosé-Hoover (NH) type thermostat is considered for Molecular Dynamics (MD) simulations of confined systems. This is based on a generalised velocity of the same generic form as the NH thermostat of Allen and Schmid, [Mol. Sim. 33, 21 (2007)]. An unthermostatted confined region is sandwiched between two walls which are thermostatted. No external shearing is imposed. Non-equilibrium Molecular Dynamics (NEMD) simulations were carried out of the time evolution of the wall and confined region temperature after a jump in temperature of the walls. Relaxation of the confined region temperature to the target value was found to be typically slower than that of the wall. An analysis of the system parameter dependence of the lag time, $\tau$, and departures from what would be expected from Fourier's law suggest that a boundary transmission heat flux bottleneck is a significant factor in the time delay. This delayed thermal equilibration would therefore become an important factor when a time-dependent (e.g., oscillatory) temperature or shearing of the walls is implemented using NEMD. Adjustments between the response time of the wall thermostat should be made compatible with that of the rest of the system, to minimise its effects on the observed behaviour.
\end{abstract}

Key words: confined liquids, thermostatting

\section{INTRODUCTION}

Temperature control or 'thermostatting' is a key technical tool in Molecular Dynamics (MD) simulations, and significant effort has been devoted to this field over recent decades. This is especially the case in Non-equilibrium Molecular Dynamics (NEMD), where the application of an externally imposed perturbation results in the system heating up. A thermostat must be applied to achieve a steady state. The NoséHoover (NH) thermostat [1-3] gives the canonical distribution of particle positions and momenta from continuously variable deterministic and time-reversible trajectories. It has proved to be one of the most popular constant temperature methods employed in equilibrium MD and NEMD simulations. The desired system temperature is achieved by a feedback mechanism which incorporates an additional dynamical or friction coefficient type variable. The fluctuations of this variable are driven by the difference between the instantaneous kinetic temperature (defined through the instantaneous kinetic energy) and the target temperature.

There are many modifications and generalisations of the Nosé-Hoover scheme in the literature [4-9]. In particular since the seminal paper by Rugh [10], different measures of temperature have been considered as the basis of alternative thermostatting schemes. In fact, there are many phase 
functions whose average at equilibrium leads to the system temperature [11, 12],

$$
k_{B} T=\frac{\langle\nabla H \cdot \mathbf{B}(\boldsymbol{\Gamma})\rangle}{\langle\nabla \cdot \mathbf{B}(\boldsymbol{\Gamma})\rangle},
$$

where $\mathbf{B}$ is a general vector field and $\boldsymbol{\Gamma}=\left(\mathbf{q}_{1}, \ldots, \mathbf{q}_{N}\right.$, $\left.\mathbf{p}_{1}, \ldots, \mathbf{p}_{N}\right)$. Alternative measures of temperature can be obtained by different choices of the vector field, $\mathbf{B}(\boldsymbol{\Gamma})$. For example, $\mathbf{B}=\left(0, \ldots, 0, \mathbf{p}_{1}, \ldots, \mathbf{p}_{N}\right)$ gives the conventional kinetic temperature used in the original $\mathrm{NH}$ thermostat, and $\mathbf{B}=\left(\mathbf{F}_{1}, \ldots, \mathbf{F}_{N}\right)$ gives an example of the so-called 'configurational' temperature, $1 / k_{B} T_{\text {conF }}=\left\langle-\sum_{i} \nabla_{i}\right.$ $\left.\cdot \mathbf{F}_{i}\right\rangle /\left\langle\sum_{i} \mathbf{F}_{i}^{2}\right\rangle$, where $\mathbf{F}_{i}=-\nabla_{\mathbf{r}_{i}} U$ is the force acting on particle $i$, and $U$ is the potential energy of the system. A variant of the $\mathrm{NH}$ thermostat based on the configurational temperature has been developed [13].

Another type of Nosé-Hoover thermostat is the Galileaninvariant thermostat, which acts on pairs of particles rather than single particles $[14,15]$. A deterministic, Galileaninvariant thermostat based on relative velocities was derived by Allen and Schmid [14], which we refer to as 'NHASv'. This can be expressed by the following equations of motion,

$$
\begin{gathered}
\frac{d \mathbf{r}_{i}}{d t}=\frac{\mathbf{p}_{i}}{m_{i}}, \\
\frac{d \mathbf{p}_{i}}{d t}=\mathbf{F}_{i}-\zeta \mathcal{V}_{i}, \\
\frac{d \zeta}{d t}=\frac{1}{Q_{\zeta}} \sum_{i}^{N}\left[\frac{\mathbf{p}_{i}}{m_{i}} \cdot \mathcal{V}_{i}-k_{B} T \nabla_{\mathbf{p}_{i}} \cdot \mathcal{V}_{i}\right],
\end{gathered}
$$

where $\zeta$ is a dynamical variable or friction-like coefficient. The constant, $Q_{\zeta}$ as in the original Nosé-Hoover method is a thermostat 'mass' parameter whose magnitude determines the extent of coupling of the thermostat to the system. In the above extension of the $\mathrm{NH}$ approach, the quantity, $\mathcal{V}_{i}$ is a function of $(\mathbf{r}, \mathbf{p})$ which can be considered to be a generalised particle velocity. In the special case, $\mathcal{V}_{i}(\mathbf{r}, \mathbf{p}) \equiv \mathbf{p}_{i}$ the formulas in Eqs. (2-4) reduce to the $\mathrm{NH}$ scheme. Averaging of the last or the thermostatting equation, Eq. (4) yields the expression for the controlled temperature, $T_{K \mathcal{V}}$,

$$
k_{B} T_{K \mathcal{V}}=\frac{\left\langle\sum_{i}^{N} \frac{\mathbf{p}_{i}}{m_{i}} \cdot \mathcal{V}_{i}\right\rangle}{\left\langle\sum_{i}^{N} \nabla_{\mathbf{p}_{i}} \cdot \mathcal{V}_{i}\right\rangle},
$$

which follows from the general definition of temperature in Eq. (1) if $\mathbf{B}(\boldsymbol{\Gamma})$ is set to $\left(0,0, \ldots, 0, \mathcal{V}_{1}, \mathcal{V}_{2}, \ldots, \mathcal{V}_{N}\right)$. In the NHASv approach the generalised velocity is expressed in the pairwise additive form, $\mathcal{V}_{i}=\sum_{j \neq i} \mathcal{V}_{i j}$. Also, $\mathcal{V}_{i j}=$ $-\mathcal{V}_{j i}$, where, $\mathcal{V}_{i j}=\left(\mathbf{v}_{i j} \cdot \mathbf{W}_{i j}\right) \mathbf{W}_{i j}=W_{i j}^{2}\left(\mathbf{v}_{i j} \cdot \hat{\mathbf{r}}_{i j}\right) \hat{\mathbf{r}}_{i j}$, $\mathbf{v}_{i j}=\mathbf{v}_{i}-\mathbf{v}_{j}$, and $\mathbf{v}_{i}=\mathbf{p}_{i} / m_{i}$ is a particle velocity. $W_{i j}$ is a function of the distance between particles $i$ and $j$. We note that the NHASv thermostat can be considered to be a special case of the generalised Nosé-Hoover equations discussed by Kusnezov et al. [16]. More details on the NHASv thermostat and its configurational temperature counterpart are given in our publication [15].

In the case of confined systems, for example a system with a slit geometry, the direct application of the above known 'bulk' thermostats may not be straightforward due to the system's geometrical constraints, and density and other system property inhomogeneities. Further modifications to the thermostatting methodology and implementation may be required. Also, in the slit geometry case it is now widely recognised that the thermostat should not be applied to the entire system but only to its wall parts. Based on current knowledge, it is clear that thermostatting schemes designed specifically for a confined MD system that are physically realistic is a topic worthy of further investigation. In this study the issue of temperature control of a system with a slab geometry is considered, and a suitable NHASv-like scheme is developed. The scheme is presented in Sec. II and its performance is tested in Sec. III. The focus of attention here is on the thermal relaxation time between the wall temperature and that of the confined part of the system.

\section{NH TEMPERATURE CONTROL OF A CONFINED SYSTEM}

A schematic diagram of the confined system of slit geometry which we employed is shown in Fig. 1. A slit pore of infinite lateral extent could be created by applying periodic boundary conditions in only the $x$ - and $y$-directions, although here we take it to be periodic in the $z$-direction as well (see below).

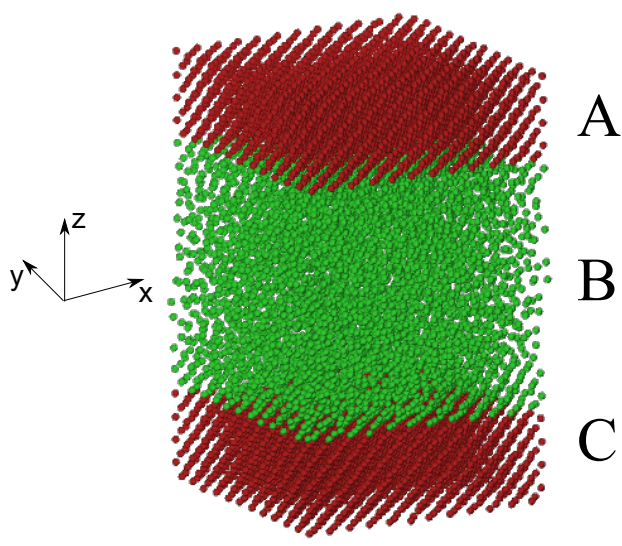

Fig. 1. Schematic representation of the confined system used in the simulations. Regions A and C are solid walls which confine the

liquid in region $\mathrm{B}$. The system is periodic in the $z$-direction

Regions $\mathrm{A}$ and $\mathrm{C}$ represent the solid walls, and region $\mathrm{B}$ is the confined part of the system, which can be a liquid, a solid or a nonequilibrium steady state if a shear is imposed. 
The total energy of the entire system can be expressed as $\mathcal{H}(\mathbf{r}, \mathbf{p})=K^{A}+K^{B}+K^{C}+U^{A A}+U^{A B}+U^{A C}+$ $U^{B B}+U^{B C}+U^{C C}$, where the symbols $K$ and $U$ denote the kinetic and potential energy, respectively, within or between regions indicated by the superscripts.

The slit geometry is a reasonable approximation for many experimental conditions in diverse fields, such as tribology (particularly elastohydrodynamic contacts) and high throughput chemical devices and MEMS devices [17-20]. Also there is increasing interest in liquids confined in nanosized geometries, either at equilibrium or in flow, where the gap between the walls need only be several molecules across. Thus, the system configuration represented in Fig. 1 can be considered to be relevant to a wide range of applications. In many respects, there can be considerable differences between the behaviour of systems in the bulk and in confinement. The boundary walls influence the confined part of the system by reducing its symmetry and causing physical property inhomogeneity in the direction perpendicular to the walls. To study a confined system most realistically the solid walls are best modelled using an atomistic description.

The way in which the wall is constructed can affect the material and dynamical properties of the modelled system. In particular, it as has been shown by Bernardi et. al. [21], that rigid walls with frozen wall atoms should be avoided. Often simulations are carried out using a tethering potential which acts as a series of 'springs' to constrain the wall atoms to vibrate about their lattice sites [18, 22-24]. Such an approach introduces extra parameters such as the stiffness of the tethering spring to the wall atoms. Also, the tethering acts as an external force on the whole system and leads to the nonconservation of the total momentum. Alternatively, the wall can be built by introducing a stronger binding energy between the wall atoms than between the confined particles [25]. This obviates the need to have a tethering potential. All those approaches have advantages and disadvantages but the last construction procedure may be considered to be the most realistic and amenable to statistical mechanical analysis, and is therefore adopted in this study.

The issue of temperature control or equivalently the 'thermostatting' of a confined system in molecular dynamics simulation has been the subject of many publications because of its importance. This has been mainly in the context of confined liquid flow, caused either by an internal pressure gradient (Poiseuille flow) or by the relative sliding of the confining walls (Couette flow). Heat is generated during such a process which must be 'extracted' from the system to allow it to reach a steady state. There are a number of options. In the confined system (assuming here for it to be a fluid) the thermostat could be applied to, (a) the wall particles only, (b) the fluid particles only or (c) both the wall and fluid particles. Recent studies have shown that the first option i.e., thermostatting only the wall particles is the most physically realistic procedure $[21,25,26]$. Also, in the case of the $\mathrm{NH}$ thermostat the conservation of total linear momentum and a zero initial value is required, which should be introduced in the confined geometry treatment (this aspect has attracted little attention in the literature). Different thermostatting strategies have been proposed and implemented in studies of confined systems [26]. New methods have been developed recently [27]. The subject is still worth further investigation, and we make a contribution to this field in this report.

In this work we show that the NHASv generalised velocity innovation can also be used to control the temperature of confined systems in a slit geometry but using a different form for the generalised velocity. We consider a sudden temperature jump in the target temperature of the walls to test the thermal equilibration behaviour throughout the whole system. This is without imposed shear or pressure induced flow in the central region (B). The following NHASv-like equations control the temperature and enforce momentum conservation of the system of slab geometry.

$$
\begin{aligned}
& \text { For region } \mathrm{A} \text {, and } i=1, \ldots, N_{A}, \\
& \qquad \begin{array}{c}
\frac{d \mathbf{r}_{i}^{A}}{d t}=\mathbf{p}_{i}^{A} / m_{i}^{A}, \\
\frac{d \mathbf{p}_{i}^{A}}{d t}=-\frac{\partial\left(U_{A A}+U_{A B}+U_{A C}\right)}{\partial \mathbf{r}_{i}^{A}}-\zeta_{A} \mathcal{V}_{i}^{A}, \\
\frac{d \zeta_{A}}{d t}=\frac{1}{Q_{A}}\left[\sum_{i=1}^{N_{A}} \frac{\mathbf{p}_{i}^{A} \cdot \mathcal{V}_{i}^{A}}{m_{i}}-g_{A} k_{B} T\right]
\end{array}
\end{aligned}
$$

for region $\mathrm{B}$, and $k=1, \ldots, N_{B}$,

$$
\begin{gathered}
\frac{d \mathbf{r}_{k}^{B}}{d t}=\mathbf{p}_{k}^{B} / m_{k}^{B} \\
\frac{\mathbf{p}_{k}^{B}}{d t}=-\frac{\partial\left(U_{B B}+U_{B A}+U_{B C}\right)}{\partial \mathbf{r}_{k}^{B}}
\end{gathered}
$$

for region $\mathrm{C}$, where $l=1, \ldots, N_{C}$,

$$
\begin{gathered}
\frac{d \mathbf{r}_{l}^{C}}{d t}=\mathbf{p}_{l}^{C} / m_{l}^{C}, \\
\frac{d \mathbf{p}_{l}^{C}}{d t}=-\frac{\partial\left(U_{C C}+U_{B C}+U_{A C}\right)}{\partial \mathbf{r}_{l}^{C}}-\zeta_{C} \mathcal{V}_{l}^{C}, \\
\frac{d \zeta_{C}}{d t}=\frac{1}{Q_{C}}\left[\sum_{l=1}^{N_{C}} \frac{\mathbf{p}_{l}^{C} \cdot \mathcal{V}_{l}^{C}}{m_{l}}-g_{C} k_{B} T\right]
\end{gathered}
$$

where $\mathcal{V}_{i}^{A}=\mathbf{p}_{i}^{A}-\frac{1}{N_{A}} \sum_{j=1}^{N_{A}} \mathbf{p}_{j}^{A}$ and $\mathcal{V}_{i}^{C}=\mathbf{p}_{i}^{C}-$ $\frac{1}{N_{C}} \sum_{j=1}^{N_{C}} \mathbf{p}_{j}^{C}$ has the form of momentum relative to that of the wall. Note that now the generalised particle velocity $\mathcal{V}_{i}^{A}$ (and $\mathcal{V}_{i}^{C}$ ) depends of the momenta of all the particles in the slab, $\left(\mathbf{p}_{1}, \mathbf{p}_{2}, \ldots, \mathbf{p}_{N_{A}}\right)$, and its form is not the sum of pairwise additive terms as in the AS scheme. The other quantities are, $g_{A}=\sum_{i=1}^{N_{A}} \nabla_{\mathbf{p}_{i}} \cdot \mathcal{V}_{i}^{A}=3\left(N_{A}-1\right)$ and $g_{C}=\sum_{i=1}^{N_{C}} \nabla_{\mathbf{p}_{i}} \cdot \mathcal{V}_{i}^{C}=3\left(N_{C}-1\right)$. The thermostat 'mass' for the two regions are $Q_{A}$ and $Q_{C}$. A straightforward derivation shows that the total linear momentum of the entire system 
is a conserved quantity without any additional conditions, i.e., $\dot{\mathbf{P}}=\dot{\mathbf{P}}_{A}+\dot{\mathbf{P}}_{B}+\dot{\mathbf{P}}_{C}=0$, where $\mathbf{P}_{X}$ denotes the momentum in the region, $X$. As in the case of the original NH scheme, a supplementary equation can be added to the equations of motion which defines the following 'energy'-like quantity,

$$
\begin{aligned}
H & =\mathcal{H}(\mathbf{r}, \mathbf{p})+\frac{1}{2} Q_{A} \zeta_{A}^{2}+g_{A} k_{B} T \ln \left(s_{A}\right) \\
& +\frac{1}{2} Q_{C} \zeta_{C}^{2}+g_{C} k_{B} T \ln \left(s_{C}\right),
\end{aligned}
$$

which is a constant of motion. The subsidiary equations for the Eqs. (6) to (8) have the simple form $\dot{s}_{A}=s_{A} \zeta_{A}$, $\dot{s}_{C}=s_{C} \zeta_{C}$.

Importantly, the proposed equations of motion generate the canonical distribution for particle momenta and positions, which can be shown by demonstrating that the density distribution $\rho=\rho\left(\mathbf{r}, \mathbf{p}, \zeta_{A}, \zeta_{C}\right)=$ $\exp (-\beta \mathcal{H}(\mathbf{r}, \mathbf{p})) \quad \exp \left(-\beta Q_{A} \zeta_{A}^{2} / 2\right) \quad \exp \left(-\beta Q_{C} \zeta_{C}^{2} / 2\right)$ (where $\beta=1 / k_{B} T$ ) is a stationary solution of Liouville's equation.

In what follows we refer to the equations of motion presented in Eqs. (6) to (8) as the 'NHASs' thermostatting method.

\section{BENCHMARKING THE BEHAVIOUR OF THE NHASS THERMOSTAT}

In order to assess the performance of the NHASs thermostat simulations on a simple test example of the confined system geometry represented in Fig. 1 have been performed. The studied system was periodic in the $x$ (streamwise) and $y$ (spanwise) directions and the contents of the middle of the cell were bounded by the walls in the $z$-direction. Each wall was composed of four layers of atoms, and the entire simulation cell was periodic in $z$-direction as well. This additional periodicity aspect ensured that the average density of the system remained constant with time. The pair interactions between the atoms in the confined region were formed from a generalised Lennard-Jones (LJ) potential, $u(r)=a \epsilon\left[(\sigma / r)^{12}-c(\sigma / r)^{6}\right]$. The chosen parameters, $a=4, c=1, \epsilon / k_{B}=120 \mathrm{~K}$ and $\sigma=0.340 \mathrm{~nm}$ correspond to the literature values for argon. The potential and force were truncated at a pair separation of $2.5 \sigma$ without any spline tapering up to that distance. The same interaction was used between the confined and wall atoms. The quantity, $c$, is often referred to as the 'wetting' parameter, and this controls the affinity of the confined atoms for the walls. Stable FCC solid walls (i.e., regions A and C) were introduced by using a stronger binding energy between the wall atoms than the confined system atoms. The LJ potential between the walls atoms took the value, $a=8$, while the other interactions in the system employed $a=4$. The results are given, unless stated, in reduced units of $\sigma$ for length, and $\left(m \sigma^{2} / k_{B} T\right)^{1 / 2}$ for time, where $k_{B}$ is Boltzmann's constant. Energy is in units of $\epsilon$ and the atomic mass, $m$, is the unit of mass. The equations of motion were integrated with the Verlet leapfrog algorithm [28] using a time step of 0.005 . The system consisted of $N=N_{A}+N_{B}+N_{C}$ atoms in total, where $N_{A}=N_{C}=576$ are for the walls, and $N_{B}=1440$ for the confined region B (see the slab geometry annotation given on Fig. 1). A range of $L$ was considered to explore the effects of aspect ratio of the simulation cell on the thermal relaxation. The cell sidelength in the $x$ - and $y$-directions was kept constant and the number of layers was increased in the $z$-direction. The values of $N$ increased through $1728,1872,2016,2160,2304,2448$ and 2592 , in order to maintain a constant average density in the central region.

In practice, for the NH-type thermostats, appropriate values of the thermostat mass can be established by performing preliminary simulations and finding a range where the distribution function of the dynamical variable is gaussian to a good approximation [29, 30]. The known dependence of $Q_{\zeta} \sim T$ and $Q_{\zeta} \sim N$ in the case of the NH thermostat $[31,32]$ was also exploited. In the calculations, $Q_{A}$ and $Q_{C}$ in the range 400-800 were used.

An effective thermostatting scheme must direct the system to a prespecified temperature and respond as quickly as possible to a change in this temperature. The calculated temperature should follow closely the target temperature and any changes in it without any spurious trends or long-lived oscillations. These apply to the wall atoms in the first instance, but in addition, in the present confined system geometry, the ability of the thermostating scheme to produce and maintain the targetted temperature in the confined B-region is also important. This feature of wall-implemented thermostats has been little studied as far as we are aware. In order to explore how well the thermostats perform in these respects, Fig. 2 shows the response of the system as a function of time to a sudden temperature increase from $T_{1}=1$ to $T_{2}=1.5$. The temperature of each region is shown in the figure.

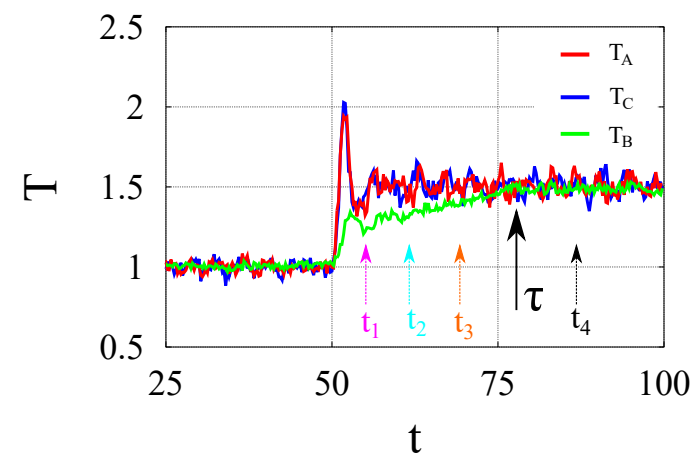

Fig. 2. The time evolution of temperature in regions A (red), B (green) and $\mathrm{C}$ (blue). The target temperature was changed from $T_{1}=1$ to $T_{2}=1.5$ at $t=50$. The relaxation time, $\tau$, pointed at by the solid arrow is the elapsed time required to achieve the target temperature in the central region. The smaller arrows, $t_{1}, t_{2}, t_{3}$ and $t_{4}$ are the times at which temperature profiles plotted in Fig. 3 are derived 
Fig. 2 shows that the calculated temperature of the walls responds relatively quickly to the new target value. There are small oscillations in the instantaneous temperature trace just after the temperature step, which is a well known feature of the NH thermostat [15]. Significantly, the temperature of the confined part of the system eventually achieves the target temperature. As may be seen in the figure, thermalisation of the liquid molecules takes a longer time than that of the wall atoms. A period of time, $\tau$, is needed for the central region molecules to converge to the new wall target temperature.

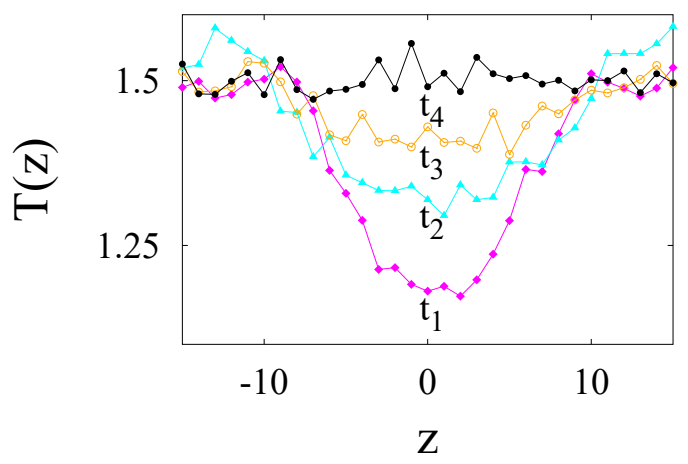

Fig. 3. Temperature profiles at times $t_{1}$ (squares), $t_{2}$ (triangles), $t_{3}$ (circles) and $t_{4}$ (dots), which are indicated by small arrows on Fig. 2

Fig. 3 shows the temperature distribution across the confined part (B-region) at several times. As may be seen, the region $\mathrm{B}$ average temperature evolves in a gradual manner towards the equilibrium flat profile. For $t<\tau$, the temperature of the walls is greater than in the central region. For a considerable time, $\tau$, after the imposition of the wall temperature jump, the temperature profile of the confined fluid is thermally inhomogeneous. This transient time is significant and depends on various system paremeters, such as the magnitude of the step in temperature, i.e., $\Delta T=T_{2}-T_{1}$, the width of the confined region $L$, and the value of the wetting parameter, $c$. These trends are illustrated in Fig. 4, where the dependence of $\tau$ on $\Delta T, L$ and $c$ is shown in frames (a), (b) and (c), respectively. It may be seen that $\tau$ increases with $\Delta T$, the film thickness $L$, and increases dramatically with decreasing affinity of the confined atoms for the walls i.e., for smaller $c$ parameter values. In regard to the $c$-dependence of $\tau$, in the $c \rightarrow 0$ limit there is no attraction between the wall and central region atoms and it can be seen in Fig. 4 that the rate of thermalisation slows down significantly in this limit. From the test data it is possible to conclude, that for the range of $\Delta T$, we have explored, $\tau \sim P(L) \Delta T / c^{\alpha}$, where $\alpha$ is close to $1 / 3$ and $P(L)$ is a second order polynomial in $L$.

The fact that the walls are thermostatted in a way which produces a rapid response means that they have a very high effective thermal conductivity compared to that of the central region. This would probably still be the case even if there were a solid in the confined region, which would have the additional lattice or phonon heat transfer mechanism to produce a uniform temperature throughout the system. This would reduce $\tau$ but it probably would still take noticeably longer than the time needed to achieve the targetted temperature in the walls (to which the thermostat is applied).
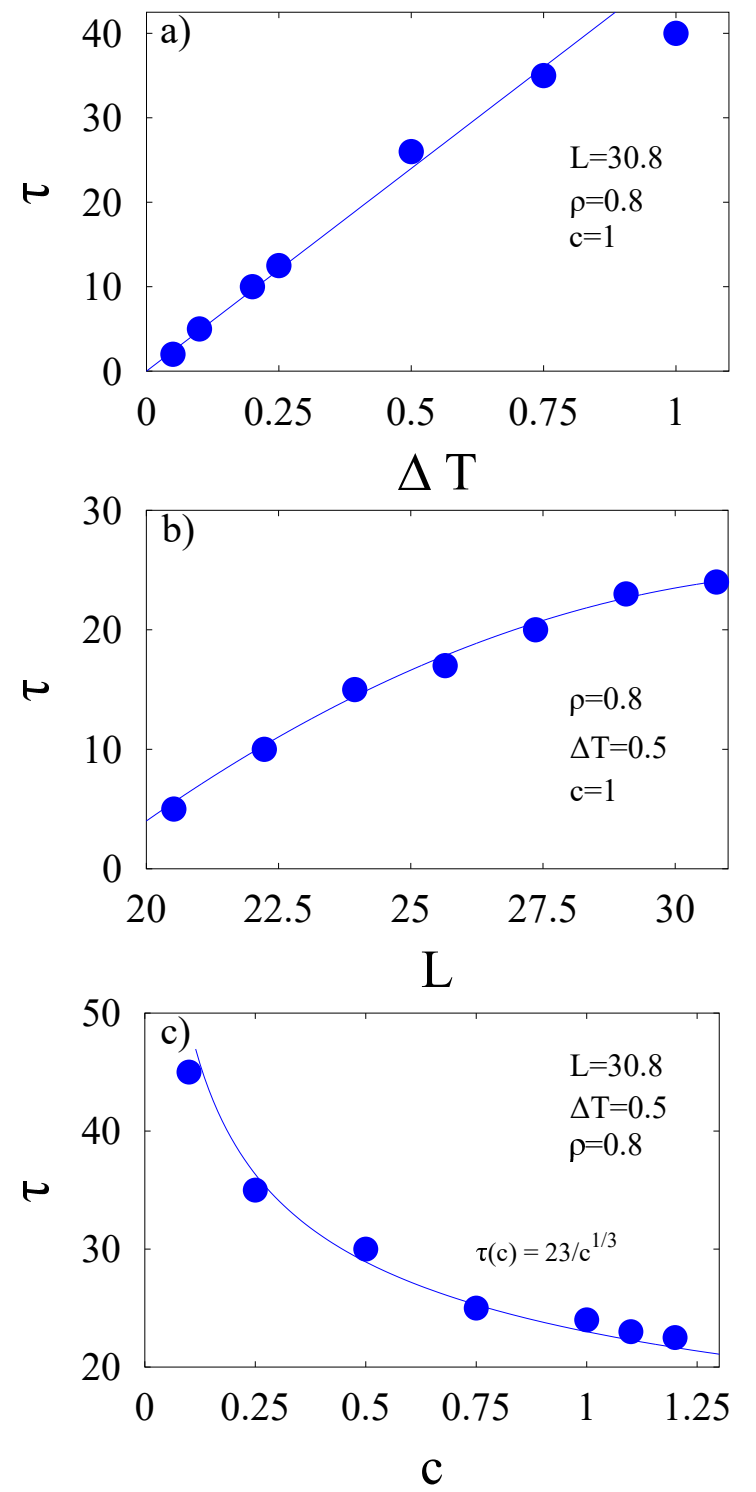

Fig. 4. The figure shows as solid dots the relaxation time, $\tau$, needed to obtain the target temperature in the system's central zone (region B) after an imposed increase in wall temperature. The quantities, $\Delta T, \rho, L$, and $c$ denote the temperature change, density of the system, vertical dimension of the system and the Lennard-Jones wetting parameter, respectively. All quantities are given using the usual Lennard-Jones reduced units convention. Panel (a) shows $\tau$ vs $\Delta T$ for $\rho=0.8, L=30.8$ and $c=1$. The solid line indicates the range where approximately linear dependence is evident. Panel (b) shows the influence of the vertical size of the system in the central zone, $L$ for $\rho=0.8, \Delta T=0.5$ and $c=1$. The solid line represents a second order polynomial fit, $\tau=-0.115 L^{2}+7.7 L-104$. The bottom panel, (c) shows the influence of the wetting parameter, $c$, for $L=30.8, \Delta T=0.5$ and $\rho=0.8$. The dependence can be fitted by $\tau(c)=24 \cdot c^{-1 / 3}$, which is given on the figure as a solid line 
Fig. 4(a) shows that the thermal relaxation time, $\tau$, depends strongly and linearly on the initial temperature difference, $\Delta T$. If Newton's law of cooling and Fourier's law ('classical laws') applied to the confined region were the only factors, then one might expect that $\tau$ would be relatively weakly dependent on $\Delta T$, all other parameters remaining the same. The trends in Fig. 4(a) therefore suggests that the thermal transmission ('Kapitza thermal resistance') across the liquid-wall interface acts as a heat flow 'bottleneck' and is a significant factor in determining the mechanism and time scale of temperature equilibration throughout the system. This dependence is also manifest in Fig. 4 (c) which shows that the wetting factor, $c$, has a dramatic effect on $\tau$. Fig. 4(b) reveals that $\tau$ increases with the confined region thickness. This aspect of behaviour would be expected from the classical laws of heat conduction and cooling as the mean temperature gradient (and hence heat flux) in the central region decreases with increasing gap width.

From this study it can be concluded that thermal equilibration in a molecularly thin unsheared system can exhibit significant differences from that predicted by the classical macroscopic expressions, and will be sensitive to the molecular structure of the wall and nature of the interactions between the atoms in the different regions. Experimental investigation of such small dimension effects are difficult to achieve with current technology, and NEMD is an ideal tool to explore these issues.

\section{CONCLUSIONS}

The new type of Nosé-Hoover (NH) thermostat, NHASs, presented in this work extends the methodology of bulk system deterministic equations of motion for generating a canonical distribution to a confined or slab geometry system. We have established here that the scheme in Eqs. (A6-A8) in Ref. [25] has the form of the Allen-Schmid version of the NH thermostat with another formula for the generalised velocity. It is not pairwise additive, but for each wall atom still relies on the velocities of all the other atoms in the wall.

A little studied aspect of the thermalisation of a confined system via bounding walls is revealed here. A transient time period, $\tau$, is required to develop the targetted temperature in a confined liquid, which can be quite substantial on a molecular timescale. The dependence of $\tau$ on several system parameters has been established, as shown in Fig. 4 . No shearing was applied to the system, so it was in a nonequilibrium state by virtue of the fact that the temperature takes time to become uniform across the wall and confined regions. This time delay follows from the atomic nature of the system, and is compounded by the usual bulk-type MD thermostats used on the walls as they have a characteristic relatively short response time. The results suggest preliminary simulations may be required in non-equilibrium molecular dynamics simulations to ascertain the natural time scale to achieve a uniform temperature distribution throughout the system for a particular set of system parameters. The response time of the wall thermostat could then be adjusted to match the natural time scale of temperature equilibration between wall and confined region. The main role of the wall thermostat is to represent the thermal transmission properties of the 'missing' wall atoms outside the slab, and should not simply be imposed without consideration of the natural thermal response time of the system as a whole. The NHASs thermostat may be applied to a confined system under shear and under external load conditions using NEMD as already anticipated in Ref. [25]. These results could be relevant to specify the parameter values and thermostatting methodology where confined film NEMD simulations are carried out to mimic a time-dependent external force, such as oscillatory shear or a time-dependent imposed temperature. The spatial and time-dependent temperature equilibration behaviour will affect significantly the properties and dynamical behaviour of the confined region.

\section{Acknowledgments}

It is a great pleasure to contribute an article to this special issue of CMST which celebrates the work of Professor W.G. Hoover, and accompanies a conference held in Sheffield, UK, on the occasion of his 80th birthday. We are happy to thank Bill Hoover for the many stimulating scientific discussions we have had with him over the years.

\section{References}

[1] W.G. Hoover, Computational Statistical Mechanics (Elsevier, Amsterdam, 1991).

[2] S. Nosé, A Molecular Dynamics Method for Simulations in the Canonical Ensemble, Mol. Phys. 52, 255 (1984).

[3] W.G. Hoover, Canonical dynamics: Equilibrium phase-space distributions, Phys. Rev. A 31, 1695 (1985).

[4] P. Hunenberger, Thermostat Algorithms for Molecular Dynamics Simulations, Adv. Polym. Sci. 173, 105 (2005).

[5] A. Bulgac, D. Kusnezov, Canonical ensemble averages from pseudomicrocanonical dynamics, Phys. Rev. A 42, 5045 (1990).

[6] G.J. Martyna, M.L. Klein, M.Tuckerman, Nosé-Hoover chains: The canonical ensemble via continuous dynamics, J. Chem. Phys. 97, 2635 (1992).

[7] W.G. Hoover, B.L. Holian, Kinetic moments method for the canonical ensemble distribution, Phys. Lett. A 211, 253 (1996).

[8] A.C. Brańka, M. Kowalik, K.W. Wojciechowski, Generalization of the Nosé-Hoover approach, J. Chem. Phys. 119, 1929 (2003).

[9] A.C. Brańka, K.W. Wojciechowski, Generalization of Nosé and Nosé-Hoover isothermal dynamics, Phys. Rev. E 62, 3281 (2000).

[10] H.H. Rugh, Dynamical Approach to Temperature, Phys. Rev. Lett. 78, 772 (1997).

[11] O.G. Jepps, G. Ayton, D.J. Evans, Microscopic expressions for the thermodynamic temperature, Phys. Rev. E 62, 4757 (2000). 
[12] G. Rickayzen, J.G. Powles, Temperature in the classical microcanonical ensemble, J. Chem. Phys. 114, 4333 (2001).

[13] C. Braga, K.P. Travis, A configurational temperature NoséHoover thermostat, J. Chem. Phys. 123, 134101 (2005).

[14] M.P. Allen, F. Schmid, A thermostat for molecular dynamics of complex fluids, Mol. Simul. 33, 21 (2007).

[15] S. Pieprzyk, D.M. Heyes, Sz. Maćkowiak, A.C. Brańka, Galilean-invariant Nosé-Hoover-type thermostats, Phys. Rev. E 91, 033312 (2015).

[16] D. Kusnezov, A. Bulgac, W. Bauer, Canonical ensembles from chaos, Ann. Phys. 204, 155 (1990).

[17] C. Gattinoni, D. M. Heyes, C. D. Lorenz, D. Dini, Traction and nonequilibrium phase behavior of confined sheared liquids at high pressure, Phys. Rev. E 88, 052406 (2013).

[18] D. M. Heyes, E. R. Smith, D. Dini, H. A. Spikes, T. A. Zaki, Pressure dependence of confined liquid behavior subjected to boundary-driven shear, J. Chem. Phys. 136, 134705 (2012).

[19] C. Gattinoni, Sz. Maćkowiak, D. M. Heyes, A. C. Brańka, D. Dini, Boundary-controlled barostats for slab geometries in molecular dynamics simulations, Phys. Rev. E 90, 043302 (2014).

[20] M. Teodorescu, S. Theodossiades, H. Rahnejat, Impact dynamics of rough and surface protected MEMS gears, Tribol. Int. 42, 197 (2009).

[21] S. Bernardi, B. D. Todd D. J. Searles, Thermostating highly confined fluids, J. Chem. Phys. 132, 244706 (2010).

[22] B. D. Todd, D. J. Evans, P. J. Daivis, Pressure tensor for inhomogeneous fluids, Phys. Rev. E 52, 1627 (1995).
[23] S. Butler, P. Harrowell, Simulation of the coexistence of a shearing liquid and a strained crystal, J. Chem. Phys. 118, 4115 (2003).

[24] J. Petravic, P. Harrowell, The boundary fluctuation theory of transport coefficients in the linear-response limit, J. Chem. Phys. 124, 014103 (2006).

[25] Sz. Maćkowiak, D. M. Heyes, D. Dini, A. C. Brańka, Nonequilibrium phase behavior and friction of confined molecular films under shear: A non-equilibrium molecular dynamics study, J. Chem. Phys. 145, 164704 (2016).

[26] X. Yong, L. T. Zhang, Thermostats and thermostat strategies for molecular dynamics simulations of nanofluidics, J. Chem. Phys. 138, 084503 (2013).

[27] S. De Luca, B. D. Todd, J. S. Hansen, P. J. Daivis, A new and effective method for thermostatting confined fluids, J. Chem. Phys. 140, 054502 (2014).

[28] D. M. Heyes, The Liquid State, (John Wiley \& Sons, Chichester, 1997).

[29] B.L. Holian, A.F. Voter, R. Ravelo, Thermostatted molecular dynamics: How to avoid the Toda demon hidden in NoséHoover dynamics, Phys. Rev. E 52, 2338 (1995).

[30] G.E. Valenzuela, J.H. Saavedra, R.E. Rozasn P.G. Toledo, Analysis of energy and friction coefficient fluctuations of a Lennard-Jones liquid coupled to the Nosé-Hoover thermostat, Mol. Simul. 41, 521, (2014).

[31] S. Nosé, Constant-temperature molecular dynamics, Progress of Theoretical Physics Supplement 103, 1 (1991).

[32] A.C. Brańka, Nosé-Hoover chain method for nonequilibrium molecular dynamics simulation, Phys. Rev. E 61, 4769, (2000).
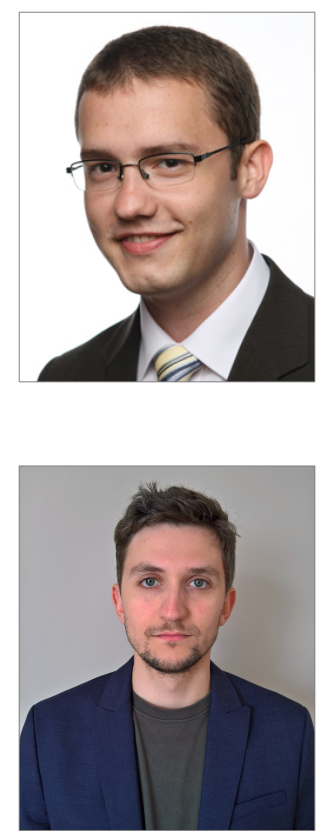

Szymon Maćkowiak was born in Wolsztyn in 1986. He studied Technical Physics at Poznań University of Technology (MSc 2010). He obtained his PhD at Poznań University of Technology in 2016 on the subject of computational investigations of tribological properties of molecular systems in a slit geometry. His scientific interests are computer simulations and friction phenomena on the micro- and nano-scale.
Sławomir Pieprzyk was born in Wolsztyn in 1986. He graduated at Poznań University of Technology and obtained his PhD in the Institute of Molecular Physics Polish Academy of Sciences in 2015. His research interests involve computer simulation methods and object-oriented programming, especially for simple liquids and soft matter. 

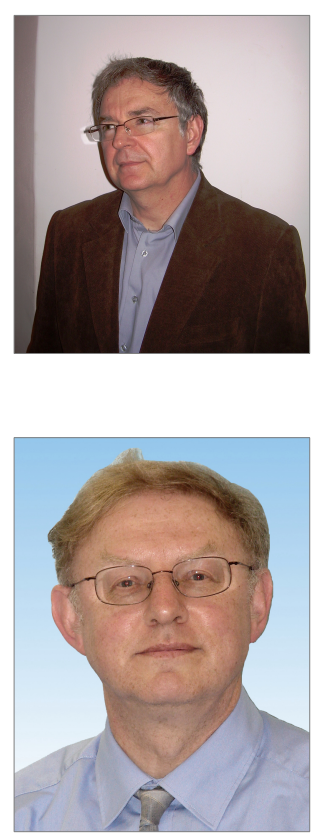

Akadiusz Brańka is associate professor at IFM PAN, and studied physics at the Adam Mickiewicz University in Poznań. He obtained his PhD in ISAS in Trieste in 1986. After his PhD he joined the Institute of Molecular Physics Polish Academy of Science, where in 2002 he received habilitation. His research interests are in the fields of structural, thermodynamics and transport properties of liquids and suspensions, liquid crystals, anomalous elasticity and simulation methods applied to particle systems.

David Heyes [PhD, University of Manchester, UK, 1977] has held postdoctoral research positions in the Vitreous State Laboratory, Department of Physics, Catholic University of America, Washington DC, USA, Department of Physical Chemistry at the University of Amsterdam, and at the Department of Chemistry, Royal Holloway, University of London. He was a Royal Society (London) 1983 University Research Fellow between 1983 and 1992 first at Royal Holloway. He is currently in the Department of Physics, Royal Holloway, University of London, and is a Principal Research Fellow in the Department of Mechanical Engineering, Imperial College London. His research interests are in the development of computer-based theoretical and modelling techniques applied to condensed matter liquid systems, principally in the areas of phase equilibria, and structural and transport properties of bulk and confined systems. 